Für kleine $t$ folgt aus Gl. (A 3)

$$
i_{\mathrm{ph}} \sim \frac{n_{0} \mathrm{I}}{\tau_{\mathrm{I}}}+\left[\frac{\varkappa n_{0} \mathrm{II}}{\tau_{\mathrm{I}} \tau_{\mathrm{II}}}-\frac{n_{0} \mathrm{I}}{\tau_{\mathrm{I}}{ }^{2}}\right] t
$$$$
\left(t \ll \tau_{\text {II }}\right),
$$

d. h. ein linearer Verlauf entsprechend

$$
i_{\mathrm{ph}}=i_{\mathrm{ph}}(0)+\text { const } \cdot t, \quad \operatorname{vgl} \text {. Abb. } 5 \text {. }
$$

(Die in Abb. 5 überlagerte sehr schnell abklingende Strahlungskomponente bleibe der Übersichtlichkeit wegen hier außer Betracht.)

Der Zeitpunkt des Strommaximums hängt in komplizierter Weise von den eingehenden Parametern ab, dagegen folgt aus Gl. (3') und $\tau_{\mathrm{I}}>\tau_{\mathrm{II}}$ unmittelbar für große $t$

$$
i_{\mathrm{ph}} \approx a \cdot \exp \left\{-t / \tau_{\mathrm{I}}\right\} \quad\left(t \gg \tau_{\mathrm{II}}\right) .
$$

In halblogarithmischer Auftragung ist der Photostrom folglich in diesem Bereich eine Gerade, aus der man leicht $\tau_{\mathrm{I}}$ und durch Extrapolation auf $t=0$ den Koeffizienten $a$ bestimmt.
Die Differenz zwischen dem extrapolierten Teil der Geraden und dem beobachteten Photostrom liefert, entsprechend

$$
a \cdot \exp \left\{-t / \tau_{\mathrm{I}}\right\}-i_{\mathrm{ph}}=b \cdot \exp \left\{-t / \tau_{\mathrm{II}}\right\},
$$

in derselben Darstellung wiederum eine Gerade, der man $\tau_{\mathrm{II}}$ und $b$ entnimmt.

Der aus der so gewonnenen Größe abgeleitete Ausdruck

$$
\frac{b}{(a-b)} \cdot \frac{\tau_{\mathrm{I}}-\tau_{\mathrm{II}}}{\tau_{\mathrm{I}}}=\frac{\varkappa n_{0} \mathrm{II}}{n_{0} \mathrm{I}}
$$

besagt, in welchem Verhältnis nachträglich und direkt angeregte Strahlung zueinander stehen. Unter der Annahme $\varkappa=1$ liefert Gl. (A 4) das Verhältnis der Besetzungszahlen der beiden Zustände zu Beginn des Nachleuchtens und damit das Verhältnis der Anregungskoeffizienten für Lawinenelektronen; denn die Anregung durch diese erfolgt in Zeiten, die sehr klein sind gegen $\tau_{\mathrm{II}}$ bzw. $\tau_{\mathrm{I}}$.

\title{
Das lonisationskontinuum von Helium, Neon und Argon*
}

\author{
Von F. J. Comes und A. Elzer \\ Institut für Physikalische Chemie der Universität Bonn \\ (Z. Naturforschg. 19 a, 721-727 [1964] ; eingegangen am 14. März 1964)
}

\begin{abstract}
Der absolute Photoionisationsquerschnitt des Edelgases Neon wurde im Energiebereich von der Ionisierungsgrenze bis $44 \mathrm{eV}(282 \AA)$ massenspektrometrisch gemessen. Die Meßresultate können qualitativ und quantitativ weitgehend mit dem von SEATon auf Grund der Dipollängenformel hergeleiteten Wirkungsquerschnitt beschrieben werden. Das Maximum des gemessenen Querschnitts wird bei $360 \AA$ mit $8,9 \cdot 10^{-18} \mathrm{~cm}^{2}$ erreicht.

Die Angabe des als absolut bezeichneten Photoionisationsquerschnitts des Neons beruht auf der Annahme, daß der von Su-Shu-Huang berechnete Wirkungsquerschnitt des Heliums dessen Ionisationskontinuum richtig beschreibt. Durch Messung der Photoionisation des Heliums kann dann aus dem Vergleich mit der Theorie die photoelektrische Ausbeute des Nickels, das als Strahlungsmonitor dient, im Bereich zwischen 500 und $280 \AA$ angegeben werden.

Mit Hilfe dieses Strahlungsdetektors wurde der Wirkungsquerschnitt des Argons gemessen, wodurch die Voraussetzung bezüglich der Heliumtheorie stark unterstützt wird. Die im Gebiet zwischen 466 und $425 \AA$ auftretenden Autoionisationsprozesse erscheinen als Minima in der Ionisationskurve, wie es in der Theorie von Fano beschrieben wird.
\end{abstract}

Die Messung der Photoionisation der leichten Edelgase Helium und Neon stößt auf größere experimentelle Schwierigkeiten. Die jeweiligen Ionisationskontinua liegen im extrem kurzwelligen Ultraviolett. Entsprechend ihrer Ionisierungsspannung von 21,56 und 24,58 eV liegt die langwellige Grenze der ionisierenden Strahlung bei 575 bzw. 504 $\AA$. Die erreichbaren Lichtintensitäten am Austrittsspalt eines Monochromators sind in diesem Wellenlängenbereich gering. Außerdem sind die Ionisierungsquerschnitte dieser Gase kleiner als $1 \cdot 10^{-17} \mathrm{~cm}^{2}$, so daß die zu messenden Ionenströme klein sind.

* F. J. Comes u. A. Elzer, Mitteilung auf der Tagung der Deutschen Bunsen-Gesellschaft in Berlin, Mai 1964.
Die massenspektrometrischen Messungen scheitern bislang daran, daß kein geeichter Photonendetektor für Wellenlängen unter $500 \AA$ existiert. Man kann sich aber eine solche Eichung verschaffen. Dazu können die Fluoreszenz von Natriumsalicylat oder der photoelektrische Effekt von Metallen ausgenutzt werden. Die Eichung geschieht in zwei Schritten. Setzt man hundertprozentige Ionisierungsausbeute voraus, dann läßt sich in einer Absorptionszelle die Zahl der absorbierten Photonen durch Messung der gebildeten Ionen bestimmen. Durch gleichzeitige Registrierung der Photonen - z. B. durch eine Messung der durch das Licht aus einer Photokathode ausgelösten Photoelektronen - kann die Ausbeute 
der Kathode gemessen werden. Eine zweite Methode beruht darauf, daß man die Messung der Photoionisation eines Gases auf den berechneten Wirkungsquerschnitt eines Gases bezieht, für das eine exakte oder wenigstens eine Theorie mit guter Näherung existiert.

Die Berechnung des Wirkungsquerschnittes für die Photoionisation von Gasen besteht in der Auffindung geeigneter, die Elektronenkonfiguration des Systems beschreibender Wellenfunktionen und die Bestimmung der Übergangsintegrale. Die exakte Lösung der Schrödinger-Gleichung ist nur für Einelektronensysteme möglich. Deshalb können auch nur für das Wasserstoffatom und wasserstoffähnliche Ionen wie $\mathrm{He}^{+}, \mathrm{Li}^{++}$usw. exakte Ausdrücke für den Wirkungsquerschnitt angegeben werden. In vereinfachter Form lautet dieser ${ }^{1}$ :

$$
\sigma=\frac{32 \pi^{2} e^{6} R Z^{4}}{3 \sqrt{3} c h^{3} v^{3} n^{5}} \cdot g
$$

( $R=$ RydberG-Konstante, $Z=$ Kernladungszahl, $v=$ Frequenz der ionisierenden Strahlung, $n=$ Hauptquantenzahl). - $g$ ist ein Faktor, der in der Nähe der Ionisierungsgrenze ohne Verlust an Genauigkeit gleich 1 gesetzt werden kann.

Aus experimentellen Gründen ist die Messung der Photoionisation dieser Teilchen sehr schwierig. Das theoretisch noch einfach zu beschreibende und gleichzeitig experimentell für eine Messung besser geeignete Atom ist das Heliumatom. Hierfür gibt es gute Näherungsfunktionen, die den Grundzustand mit zuverlässiger Genauigkeit angeben. Desgleichen vermögen die zur Berechnung des ionisierten Zustandes benutzten Funktionen diesen gut zu beschreiben. Es sind von verschiedenen Autoren Berechnungen des Frequenzverlaufs des Ionisationskontinuums von Helium durchgeführt worden, die im Zusammenhang mit zwei vorausgegangenen Messungen diskutiert werden.

Auf der Basis der Theorie von Su-Shu-Huang ${ }^{2}$ für das Heliumatom ist die photoelektrische Ausbeute des Nickels bestimmt worden. Mit Hilfe dieser Ausbeutekurve wurde der Wirkungsquerschnitt des Neons angegeben. Berechnungen des Übergangs-

1 D. R. Bates, Monthly Notices Roy. Astron. Soc. 106, 423 [1946].

2 Su-Shu-Huang, Astrophys. J. 108, 354 [1948].

3 D. R. Bates, Monthly Notices Roy. Astron. Soc. 100, 25 [1939]; 106, 432 [1946].

4 M. J. Seaton, Proc. Roy. Soc., Lond. A 208, 408 [1951]; Proc. Phys. Soc., Lond. A 67, 927 [1954]. integrals für Neon sind von Bates ${ }^{3}$ und Seaton ${ }^{4}$ vorgenommen worden. Letzterer hat sowohl die Dipollängen- als auch die Dipolgeschwindigkeitsformel benutzt. In zwei früheren Messungen ist der Wirkungsquerschnitt für die Gesamtabsorption des Neons von $\mathrm{W}_{\text {EISSLer }}{ }^{5}$ und Mitarbeitern und von Ditchburn $^{6}$ bis etwa $220 \AA$ vermessen worden. Dershem und Schein $^{7}$ bestimmten den Wirkungsquerschnitt des Neons für die Kohlenstofflinie Ka $(44,6 \AA)$ zu $0,436 \cdot 10^{-18} \mathrm{~cm}^{2}$.

Eine mit Hilfe der Nickelausbeutekurve vorgenommene Messung der Photoionisation des Argons kann durch einen Vergleich mit bestehenden Argonmessungen ebenfalls zur Prüfung der Grundannahmen (Richtigkeit des theoretischen $\sigma_{\mathrm{He}}$ ) und damit zugleich der Nickelausbeutekurve herangezogen werden. Die Úbereinstimmung der Messungen ist sehr gut. Mit Hilfe des so geeichten Photonendetektors können jetzt massenspektrometrische Messungen der Photoionisation aller geeigneten Gase bis $280 \AA$ durchgeführt werden.

\section{Meßanordnung}

Die Meßapparatur gleicht in vielen Teilen einer früher beschriebenen Anordnung ${ }^{8}$. Sie besteht aus einer Kombination von $\mathrm{S}$ e y a - N a m i o k a - Monochromator und Vierpolmassenspektrometer. Als Lichtquelle wurde eine wassergekühlte Kapillarfunkenstrecke benutzt, die bei einem Druck von einigen Zehntel Torr, einer Parallelkapazität von $0,3 \mu \mathrm{F}$ und $2 \mathrm{kV}$ Gesamtspannung mit Netzfrequenz betrieben wurde. Das Emissionsspektrum der Lichtquelle besteht bei diesen Betriebsbedingungen aus einem intensitätsreichen Linienspektrum. Die Benutzung eines Linienspektrums zur Messung der Photoionisation von Helium und Neon hat große Vorteile gegenüber der Verwendung eines Kontinuums, da bei den verwendbaren Strahlungsquellen die Lichtintensität des Linienspektrums wesentlich größer und das zu messende Ionisationskontinuum ohne Struktur ist. Die den Ionisationsraum durchsetzenden Lichtimpulse liegen im Bereich zwischen $4 \cdot 10^{9}$ und $2 \cdot 10^{6}$ Quanten pro Funke für das Spektrum zwischen 800 und $280 \AA$.

Der Hauptteil des Monochromators ist wie in der früheren Konstruktion aus Edelstahlteilen zusammengeschweißt und hat neben dem die Lichtquelle tragenden Spektrometerteil zwei Experimentierausgänge, so

\footnotetext{
5 Po Lee u. G. L. Weissler, Proc. Roy. Soc., Lond. A 220, 71 [1953].

${ }^{6}$ R.W. Ditchiurn, Proc. Phys. Soc., Lond. A 75, 461 [1960].

7 E. Dershem u. M. Schein, Phys. Rev. 37, 1238 [1931].

8 F. J. Comes U. W. Lessmans, Z. Naturforschg. 19 a, 65 [1964].
} 
daß mit einer Lichtquelle und einem optischen Gitter zwei getrennte Apparaturen zeitlich hintereinander versorgt werden können. Das Gitter hat 1200 Furchen pro $\mathrm{mm}$, ist für eine bevorzugte Wellenlänge bei senkrechtem Einfall von $800 \AA$ geritzt und hat einen Radius von $998 \mathrm{~mm}$.

Die Flugrichtung der Ionen steht senkrecht zur Richtung des Lichtbündels und zur Höhe des optischen Spaltes. Durch ein $9 \mathrm{~cm}^{2}$ großes Netz werden die Ionen aus dem Ionisationsraum herausgezogen und in einem Linsensystem auf die kreisförmige $(\phi=1,4 \mathrm{~mm})$ Eintrittsblende des Vierpolmassenspektrometers fokussiert. Das Linsensystem besteht aus 2 Zylinderlinsen und 2 Vierpollinsen. Dadurch können noch Ionen, die einen größeren Abstand von der optischen Achse des Linsensystems haben, ins Massenspektrometer gelangen.

Der Photonendetektor hat eine Nickelkathode, deren photoelektrische Ausbeute im Verlauf der Arbeit bestimmt wird. Die Photozelle ist gasdicht mit dem Ionisationsraum verbunden und so mit Spannungen versehen, daß keines der gebildeten Photoelektronen den Ionisationsraum erreichen kann. Wenn die Ionen das $0,5 \mathrm{~m}$ lange Vierpolfeld durchlaufen haben, werden sie in einem offenen Multiplier eigener Bauart registriert. Die Multiplierdynoden bestehen aus einer Silber-Magnesium-Legierung, und das System hat bei einer Spannung von 4,5 kV eine Verstärkung für die zu messenden Ionen von etwa $10^{5}$. Die Vierpolfrequenz wurde bei den folgenden Messungen auf $2 \mathrm{MHz}$ eingestellt. Die durch die Ionen und Photonen hervorgerufenen Registrierströme werden mit Schwingkondensatorverstärkern gemessen und dann auf getrennten Schreibern registriert. Durch ein Ionisationsmanometer und ein thermoelektrisches Manometer in der Ionenquellenzuleitung wird der Druck in der Ionenquelle bestimmt. Die Eichung der Manometeranzeige wird durch einen Vergleich der bei den Linien 762 und $703 \AA$ in Argon und $504 \AA$ in Helium erzielten Gesamtionenbildung in der Ionenquelle mit Literaturwerten vorgenommen, woraus der Druck des Neons berechnet werden kann. Dazu wurde für Neon der im Ionisationsraum bei der Einstrahlung der Linie $554 \AA$ entstehende Ionenstrom als Eichwert gemessen. Der Motorantrieb des Monochromators gestattet, das Lichtquellenspektrum mit einer Geschwindigkeit von $10 \AA$ pro Minute zu durchfahren.

\section{Durchführung der Messungen}

In der vorliegenden Arbeit wurde die Ionenbildung durch Photonen in Helium, Neon und Argon untersucht; gemessen werden der das Massenspektrometer durchlaufende Ionenstrom bzw. ein diesem proportionaler Sekundärelektronenstrom am Ausgang des Multipliers und der durch die Photonen aus der Nickelkathode ausgelöste Photoelektronenstrom, der eine Funktion der Photonenenergie ist. Im größten Teil des vorliegenden Wellenlängenbereiches liegen keine Ergebnisse über photoelek- trische Ausbeuten vor, so daß hier eine der in der Einleitung erwähnten Methoden zur Messung der Photoausbeuten zur Anwendung kommen muß. Eine Eichung der Kathode durch Vergleichsmessungen der Strahlungsleistung mit einem Thermoelement läßt sich wegen der zu schwachen Ausgangsleistung am Monochromatorspalt nicht durchführen.

Zur Ausbeutemessung wurde die zweite der erwähnten Methoden benutzt. Aus diesem Grunde mußte zuerst die Photoionisation des Heliums gemessen werden. Das Verhältnis des bei der Quantenenergie $h v$ gemessenen Stroms der $\mathrm{He}^{+}$-Ionen zum gleichzeitig ausgelösten Photoelektronenstrom in der Nickelphotozelle ist in Abb. 1 aufgetragen. Im Meßbereich liegen 25 Meßpunkte. Die Photonenintensi-

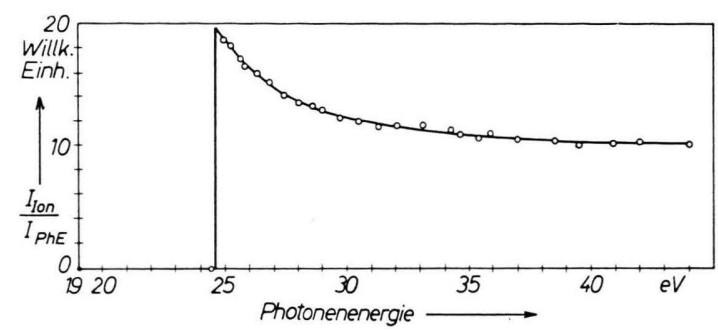

Abb. 1. Nichtkorrigierte Helium-Ionisationskurve. Verhältnis der gebildeten Ionen zu den aus der Nickelkathode ausgelösten Photoelektronen.

täten der einzelnen Linien im Ionisationsbereich des Heliums liegen zwischen $7 \cdot 10^{7}$ und $2 \cdot 10^{6}$ Quanten pro Funke. Die Meßpunkte zu beiden Seiten der Ionisationsgrenze sind 507 und $504 \AA$ A. Der spektroskopische Wert für die Grenze ist $504,3 \AA^{9}$. Aus dieser Messung wurde mit Hilfe der Theorie von $\mathrm{S}_{\mathrm{U}}-\mathrm{Shu}_{\mathrm{H}}-\mathrm{Hunag}^{2}{ }^{2}$, die, wie in der Diskussion der Meßergebnisse näher erläutert, auch durch neuere Berechnungen belegt wird, eine Kurve für die Größe und den Frequenzverlauf der photoelekrischen Ausbeute an unpräpariertem Nickel gewonnen.

Zur Berechnung der photoelektrischen Ausbeute aus der theoretischen Ionisationskurve muß der Druck im Ionisationsraum bestimmt werden. Die Eichung der Monometeranzeige geschah durch die Messung der Photoionisation des Argons bei den Wellenlängen 762 und $703 \AA$. Im Wellenlängenbereich in der Nähe der Ionisationsgrenze sind der Ionisationsquerschnitt des Argons und die Intensität der Lichtquelle groß, so daß diese Messung mit ausreichender Genauigkeit durchgeführt werden kann.

9 C. S. Moone, Atomic Energy Levels, Nat. Bur. Stand. Circ. No. 467, Vol. I, 1949. 
Hierzu wird aus dem Vergleich der gemessenen Gesamtionenbildung in der Ionenquelle mit dem Literaturwert für den Wirkungsquerschnitt der Ionenquellendruck berechnet.

Huffman, Tanaka und Larrabee ${ }^{10}$ sowie Po Lee und WeissLer ${ }^{11}$ geben für die Wellenlänge $762 \AA$ einen Wirkungsquerschnitt von 35,7 und $35,8 \mathrm{Mb}$ an. Den folgenden Messungen ist $\sigma=35,7 \mathrm{Mb} \mathrm{zu}$ grunde gelegt. Hieraus läßt sich der Argondruck in der Ionenquelle bestimmen, der bei den Messungen $4 \cdot 10^{-4}$ Torr betrug. Der bei dem gleichen Druck gemessene Querschnitt für die Wellenlänge $703 \AA$ betrug $39,2 \mathrm{Mb}$ in Übereinstimmung mit Huffuan et al. Po Lee und Weissler liegen hier um $10 \%$ niedriger und geben $35,3 \mathrm{Mb}$ an. Durch diese Messungen wird die Druckanzeige des Manometers geeicht. Sie läßt sich dann auf die anderen Gase umrechnen. Zur Kontrolle wurde ebenfalls der Heliumdruck bestimmt.

Für eine besonders intensive Linie (465 $\AA$ ) wurde, wie schon beim Argon, für Helium die Gesamtionenbildung in der Ionenquelle gemessen. Aus der genauen Kenntnis des Lichtweges und des Ionisierungsquerschnitts läßt sich bei bekannter Zahl der ionisierenden Photonen hieraus der genaue Heliumdruck bestimmen. Die Kenntnis der Photonenzahl ist zunächst nur für Energien in der Nähe der Ionisie- rungsgrenze bekannt. Über den zugrunde gelegten theoretischen Wirkungsquerschnitt kann jedoch auf diese Grenze zurückgerechnet und so der Heliumdruck bestimmt werden.

Aus der auf der Nickelausbeute beruhenden Ionisierungskurve des Heliums (Abb. 1) und deren Vergleich mit dem Verlauf des theoretischen Wirkungsquerschnittes von Huang gewinnt man die Anzahl der von einer unpräparierten Nickeloberfläche durch Photonen ausgelösten Elektronen. In Abb. 2 a ist die bis dahin bekannte Nickelausbeute wiedergegeben ${ }^{12}$. Wie die Meßkurven für die mehr oder weniger stark ausgeheizten Nickelproben zeigen, ist im Bereich unter $500 \AA$ ein Ansteigen der Ausbeute zu erkennen. Dasselbe Ergebnis bringt auch der Vergleich von Heliumkurve (Abb. 1) und Theorie. Die Kurve (Abb. 2 b) durchläuft also zunächst bei $530 \AA$ ein Minimum, steigt an und fällt im kurzwelligen Teil wieder ab. Der Abfall verläuft so, daß die Messungen der Nickelausbeute durch Lukirskir, Rumsk und Smirnov ${ }^{13}$, die diese Autoren im weichen RöntgenGebiet vorgenommen haben, wahrscheinlich durch ein drittes Maximum an die übrige Kurve angeschlossen werden können.

Nach Abschluß der Arbeit wurde eine Messung von SAmson ${ }^{14}$ bekannt, in der ebenfalls die Photoionisation von Argon gemessen wurde. Der Autor

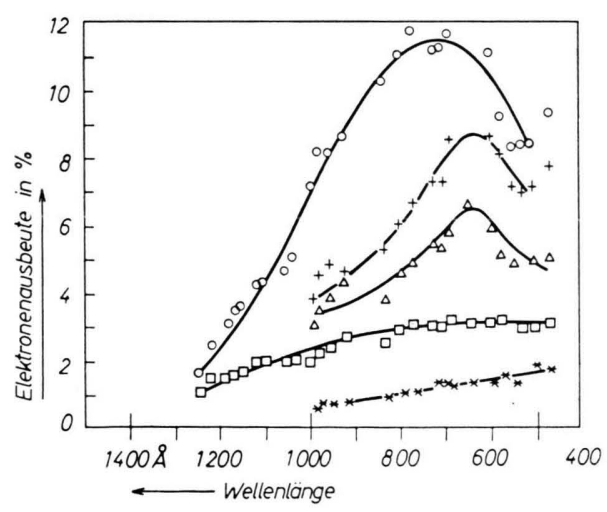

Abb. 2 a. Photoelektrische Ausbeute von Nickel nach W

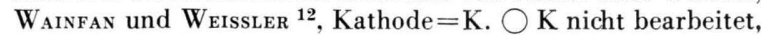
$+\mathrm{K} 1$ min lang ausgeheizt bei $5 \cdot 10^{-2}$ Torr $\mathrm{O}_{2}$ und einer Temperatur von $800^{\circ} \mathrm{C}, \triangle \mathrm{K}$ ausgeheizt, dann $\frac{1}{2}$ Stunde lang bei Zimmertemperatur einem $\mathrm{O}_{2}$-Druck von $1 \cdot 10^{-2}$ Torr aus gesetzt, $\square \mathrm{K}$ ausgeheizt bei $10^{-5}$ Torr (Restgas), $* \mathrm{~K}$ bei einer Temperatur von $900{ }^{\circ} \mathrm{C}$ und $10^{-5}$ Torr (Restgas).

10 R. E. Huffman, Y. Tanaka u. J. C. Larrabee, J. Chem. Phys. 39, 902 [1963].

11 Po Lee u. G. L. Weissler, Phys. Rev. 99, 540 [1955].

12 W. Walker, N. Wainfan u. G. L. Weissler, J. Appl. Phys. 26, 1366 [1955].

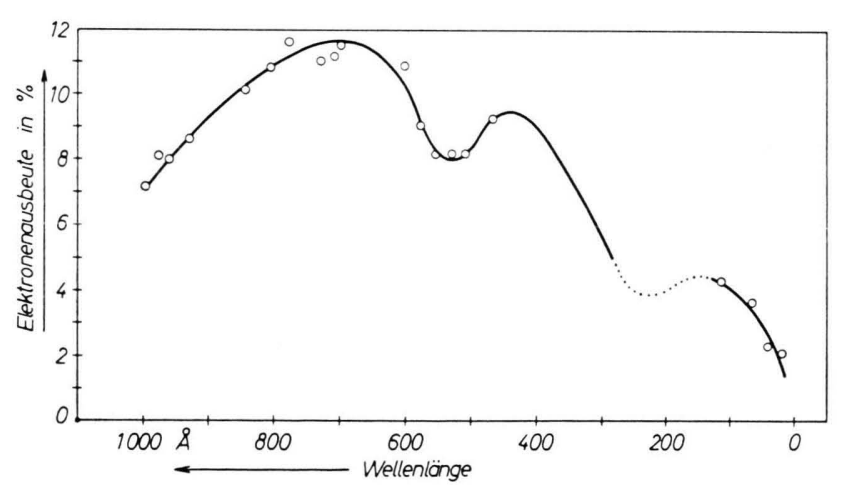

Abb. 2 b. Zusammengesetzte Kurve für die photoelektrische Ausbeute von nicht bearbeitetem Nickel.

13 A. P. Lukirskit, M. A. Rumsk u. L. A. Smirnov, Opt. Spectr. USSR 9, 265 [1960].

14 J. A. R. Samson, Phys. Rev. 132, 2122 [1963]. 
benutzte eine doppelte Ionisationskammer bei einem Argondruck von 0,05-0,5 Torr. Die Messungen erstrecken sich auf den Bereich zwischen 25 und $31 \mathrm{eV}$. Hier tritt im Argon eine zur Grenze ${ }^{2} \mathrm{~S}_{1 / 2}$ konvergierende stark verbreitete Absorptionsserie auf, wie Madden und Codling ${ }^{15}$ zeigen konnten. Die Linien sind als Minima in das Ionisationskontinuum eingebettet und entsprechen einer der in der Theorie von $\mathrm{F}_{\text {ANO }}{ }^{16}$ angegebenen Möglichkeiten für die Form der Autoionisationskurve. Der wahrscheinliche Übergang ist $3 \mathrm{~s}^{2} 3 \mathrm{p}^{6}{ }^{1} \mathrm{~S}_{0}-3 \mathrm{~s} \mathrm{p}^{6} n \mathrm{p}^{1} \mathrm{P}_{1}{ }^{0}$. In der vorliegenden Arbeit wurde dieses Phänomen ebenfalls gefunden, wie die Meßkurve in Abb. 4 zeigt. Mit Hilfe der Ausbeutekurve (Abb. 2 b) und der bei den Wellenlängen 762 und $703 \AA$ vorgenommenen Druckeichung wurde der Ionisierungsquerschnitt berechnet. Im Bereich der SAmsonschen Messungen stimmen beide Kurven sehr gut überein, wodurch sich, da beide Resultate auf verschiedenen Wegen gewonnen wurden, eine starke Stütze für das Ergebnis von Abb. $2 \mathrm{~b}$ und damit für die Richtigkeit der Heliumtheorie in diesem Bereich ergibt.

Um eine gute Druckbestimmung für Neon zu gewährleisten, wurden die Messungen an allen Edelgasen bei möglichst übereinstimmenden Drucken vorgenommen. Die Frequenzabhängigkeit der Photoionisation des Neon zeigt Abb. 3 a. Aufgetragen ist das Verhältnis der Zahl der gebildeten Ionen zu den

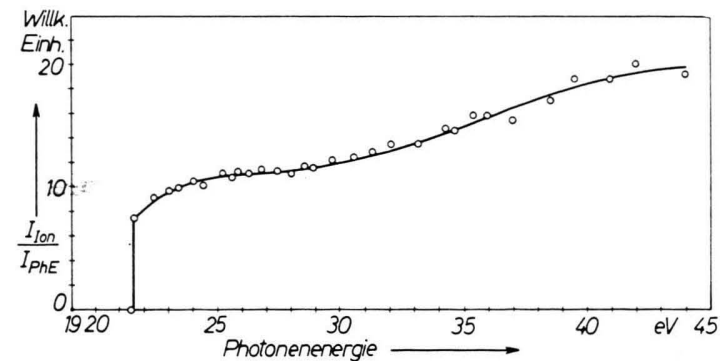

Abb. 3 a. Nichtkorrigierte Neon-Ionisationskurve. Verhältnis der gebildeten Ionen zu den aus der Nickelkathode ausgelösten Photoelektronen.

aus der Kathode ausgelösten Photoelektronen. Der stärkere Anstig der Kurve bei $30 \mathrm{eV}$ deutet auf den starken Abfall der Ausbeutekurve von Abb. 2 b hin. Mit den Zahlenwerten dieser Kurve wurde die Neonionisationskurve korrigiert und durch Berücksichtigung des Ionenquellendruckes der Ionisationsquer-

15 R. P. Madden u. K. Codling, Phys. Rev. Letters 10, 516 [1963]. schnitt des Neons gewonnen (Abb. 3 b). Der Querschnitt steigt von der Ionisierungsgrenze aus an und erreicht für $360 \AA$ ein Maximum. Ein Vergleich dieses Ergebnisses mit Messungen von WeIssler und Ditchburn sowie mit den theoretischen Werten von

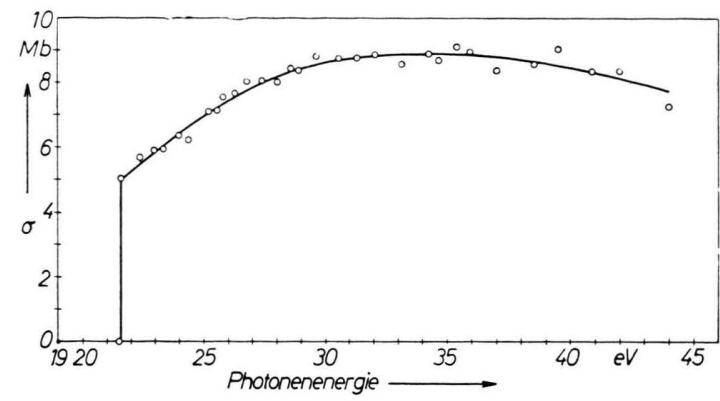

Abb. 3 b. Energieabhängigkeit des Photoionisationsquerschnittes von Neon.

SEaton vermittelt Abb. 3 c. Der hier gefundene Neonquerschnitt weicht vor allem im kurzwelligen Teil von den bisherigen experimentellen Kurven ab. Von den theoretischen Werten vermögen am ehesten die nach der Dipollängenformel gewonnenen Ergebnisse den experimentellen Befund zu approximieren.

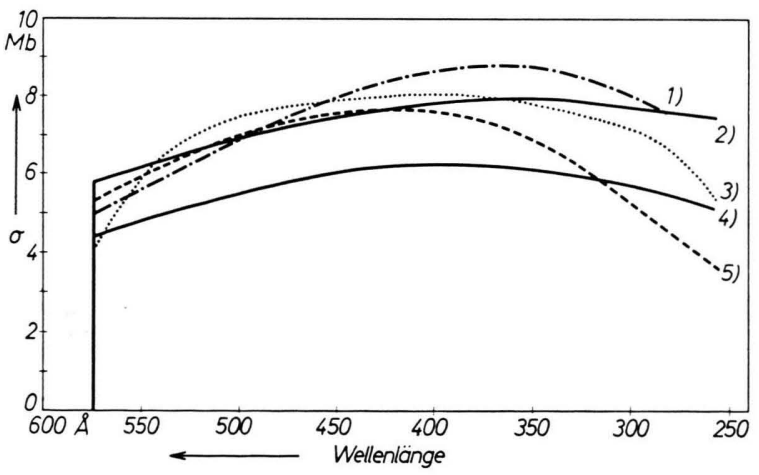

Abb. 3 c. Vergleich des Neon-Ionisationsquerschnittes nach Theorie und Experiment: 1) vorliegende Messung, 2) nach Seaton (Dipollängenformel), 3) nach Ditchburn, 4) nach Seaton (Dipolgeschwindigkeitsformel), 5) nach Po LeE und W EISSLER.

\section{Diskussion der Meßergebnisse}

Die Berechnung des Wirkungsquerschnittes für die Photoionisation setzt die Lösung des Übergangsintegrals bzw. des Matrixelements voraus. Das Ma-

16 U. Fano, Phys. Rev. 124, 1866 [1961]. 
trixelement ist von der Gestalt:

$$
\int \psi_{\mathrm{a}}{ }^{*} r \psi_{\mathrm{e}} \mathrm{d} \tau,
$$

wobei $\psi_{\mathrm{a}}$ und $\psi_{\mathrm{e}}$ die Wellenfunktionen des Anfangs(Atom im Grundzustand) und Endzustandes (Ion plus Elektron) des Systems sind, $r$ die Summe der Radienvektoren der Elektronen bedeutet und die Integration über den gesamten Konfigurationsraum zu erstrecken ist. Die Aufstellung geeigneter Näherungsfunktionen für $\psi_{\mathrm{a}}$ und $\psi_{\mathrm{e}}$ im Falle des Mehrelektronensystems, sowie die Lösung von (2) führen zur Bestimmung des Wirkungsquerschnittes. Während die Form von (2) der sogenannten Dipollängenformel entspricht, kann dieser Ausdruck für den Fall, daß $\psi_{\text {a }}$ und $\psi_{\mathrm{e}}$ exakte Lösungen der Wellengleichung des Systems sind, in zwei identische Ausdrücke umgeformt werden, die Dipolgeschwindigkeits- und Dipolbeschleunigungsformel genannt werden. Für den praktischen Fall, daß $\psi_{\mathrm{a}}$ und $\psi_{\mathrm{e}}$ Näherungsfunktionen darstellen, ist die Voraussetzung der Identität dieser drei Ausdrücke nicht mehr gegeben. Sie stellen deshalb Formeln dar, deren Brauchbarkeit für die Berechnung des Wirkungsquerschnitts von der jeweiligen Wahl der das System beschreibenden Wellenfunktionen abhängt.

Bei der Angabe des Wirkungsquerschnitts für die Photoionisation von Neon und Argon in Abb. 3 und 4 wurde die Richtigkeit der Huangschen Berechnungen des Heliumionisationskontinuums zugrunde gelegt. Schon früher hatten VINTI ${ }^{17}$ und WheeleR ${ }^{18}$ Berechnungen des Heliumkontinuums durchgeführt. Diesen und auch weiteren Arbeiten ist die Approxi-

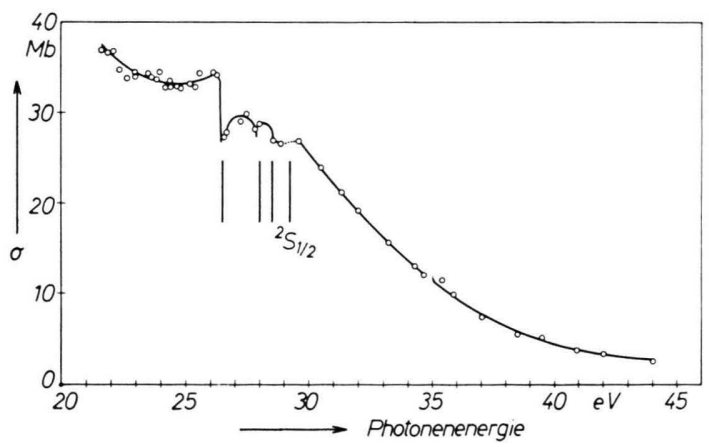

Abb. 4. Energieabhängigkeit des Photoionisationsquerschnittes von Argon im Energiebereich von 22 bis $44 \mathrm{eV}$.

17 J. P. Vinti, Phys. Rev. 44, 524 [1933].

18 J. A. Wheeler, Phys. Rev. 43, 258 [1933].

19 A. L. Stewart u. W. J. Wilkinson, Proc. Phys. Soc., Lond. A 75, 796 [1960]. mation des freien Zustandes (Ion + Elektron) durch wasserstoffähnliche Wellenfunktionen gemeinsam. In den Ansätzen für den gebundenen Zustand werden sowohl Wasserstoff-Funktionen als auch die HyLLERaAssche Funktion benutzt. Während die HyllerAas-Funktion zweifellos dem Heliumgrundzustand am besten angepaßt ist, bedingt sie jedoch eine Erschwerung der Rechnungen. Sowohl Su-Shu-Huang als auch neuere Rechnungen von Stewart und $\mathrm{W}_{\mathrm{IL}}$ Kinson ${ }^{19}$ benutzen den Hylleraas-Ansatz für den Heliumgrundzustand.

Die beiden letzten Autoren untersuchten, wie weit die Approximation des freien Zustandes durch wasserstoffähnliche Funktionen zulässig ist. Die Lösungen sind sowohl für ein Coulomb-Feld als auch für ein Hartree- und Hartree-Fock-Feld mit der Dipollängen- und Dipolgeschwindigkeitsformel durchgeführt. Die Abweichungen von den Huangschen Ergebnissen sind gering und treten im wesentlichen nur in der Nähe der Ionisierungsgrenze auf (Abb. 5).

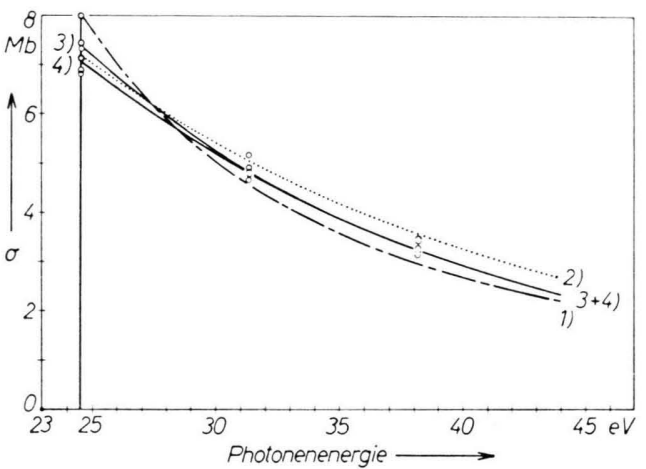

Abb. 5. Theoretischer Ionisationsquerschnitt des Heliums: 1) nach Vinti, 2) nach Wheeler, 3) nach Huang (Dipolgeschwindigkeitsformel), 4) nach Huang (Dipollängenformel). Die eingezeichneten Punkte (O) entsprechen den von Stewart und WiLKinson angegebenen Werten.

Die Übereinstimmung mit Berechnungen von BurGess und Seaton ${ }^{20}$ ist ebenfalls gut. Deshalb wurde den Heliummessungen der Mittelwert des von Huang mit der Dipollängen- und Dipolgeschwindigkeitsformel erhaltenen Wirkungsquerschnitts zugrunde gelegt. Die beiden Kurven weichen nur gering in der Nähe der Ionisierungsgrenze voneinander ab. Dieser Mittelwert stimmt innerhalb der Meßgenauigkeit

20 A. Burgess u. M. J. Seaton, Monthly Notices Roy. Astron. Soc. 120, 121 [1952]. 
mit den von Po Lee und Weissler ${ }^{11}$ sowie den im kurzwelligen Gebiet unter $300 \AA$ von Axelrod und Givens ${ }^{21}$ durchgeführten Messungen überein.

Aus dem Vergleich der Theorie mit dem experimentellen Ergebnis resultiert die Nickelausbeutekurve (Abb. 2 b). Die Kurve hat zwei, vielleicht sogar drei selektive Maxima. Durch Ausheizen läßt sich das erste Maximum beseitigen ${ }^{12}$, so daß zu vermuten ist, daß Oberflächenbelegungen für die erhöhte Ausbeute verantwortlich sind. Die unter $\mathrm{Zu}$ grundelegung dieser Nickelausbeutekurve gewonnene Ionisationskurve des Neon wurde schon in Abb. $3 \mathrm{c}$ mit früheren Experimenten und den Ergebnissen der Theorie von Seaton verglichen. Die Messungen zeigen eindeutig, daß die mit der Dipollängenformel gewonnenen Zahlenwerte mit dem experimentellen Befund am besten übereinstimmen. Beide Kurven

21 N. N. Axelrod u. M. P. Givens, Phys. Rev. 115, 97 [1959]. erreichen an der gleichen Stelle der Energieskala ihren maximalen Wert. Über den vermessenen Wertebereich stimmen sie innerhalb $10 \%$ überein.

Die Übereinstimmung des Argonquerschnittes mit dem von SAMson gemessenen Wert ist sehr gut. Nach der Theorie von Fano führt die Wechselwirkung eines diskreten autoionisierenden Zustandes mit einem Kontinuum zu asymmetrischen Peaks im Spektrum. Sie können als Maxima, Minima oder auch in gewissen Zwischenformen im Ionisationskontinuum auftreten. Im Einklang mit den Ergebnissen dieser Theorie sinkt der Querschnitt im Argonionisationskontinuum im Bereich der Autoionisation.

Wir danken Herrn Prof. Dr. W. GRoth für die ständige Förderung dieser Arbeit und der Deutschen Forschungsgemeinschaft für die Bereitstellung von Mitteln für ihre Durchführung.

\title{
Negative lonen aus Alkalihalogeniden und Elektronenaffinitäten der Alkalimetalle und Alkalihalogenide
}

\author{
Von H. Ebinghaus \\ I. Institut für Experimentalphysik, Hamburg \\ (Z. Naturforschg. 19 a, 727-732 [1964]; eingegangen am 15. Februar 1964)
}

\begin{abstract}
Die Bildung negativer Ionen aus Alkalihalogeniddämpfen durch Elektronenresonanzeinfang wurde massenspektrometrisch untersucht. Die gefundenen Ionen der Form $\mathrm{M}^{-}, \mathrm{X}^{-}, \mathrm{MX}^{-}$und $\mathrm{M}_{2} \mathrm{X}^{-}(\mathrm{M}=\mathrm{Li}$, $\mathrm{Na}, \mathrm{K}, \mathrm{Rb}, \mathrm{Cs} ; \mathrm{X}=\mathrm{F}, \mathrm{Cl}, \mathrm{Br}, \mathrm{J})$ konnten an Hand der Energiebilanzen und der Intensitätsverhältnisse der Entstehung aus monomeren und dimeren Molekülen zugeordnet werden. Aus den gemessenen Appearance-Potentialen und den bekannten Dissoziationsenergien wurden für die Elektronenaffinitäten der Alkalimetalle untere Grenzwerte bestimmt, die bei $0,5 \mathrm{eV}$ liegen, für einige Alkalihalogenide liegen sie bei $1,2 \mathrm{eV}$. Bei den Werten für die Alkalimetalle handelt es sich vermutlich um die tatsächlichen Elektronenaffinitäten.
\end{abstract}

Nachdem über die Bildung positiver Ionen durch Elektronenstoß aus Alkalihalogeniden vielfach berichtet worden ist ${ }^{1-4}$, erschien es interessant, ebenfalls die Bildung negativer Ionen aus Alkalihalogenidmolekülen zu untersuchen, zumal sich die Möglichkeit der Bestimmung der Elektronenaffinitäten der Alkalimetalle bot, für die bisher kaum experimentelle, dagegen zahlreiche theoretische Werte vorliegen. Die Experimente mit positiven Ionen ergaben, daß in Alkalihalogeniddämpfen neben monomeren auch dimere und trimere Moleküle vorhanden

1 L. Friedmann, J. Chem. Phys. 23, 477 [1955].

2 J. Berkowitz u. W. A. Chupкa, J. Chem. Phys. 29, 653 [1958]. sind, so daß auch negative Ionen aus diesen Polymeren zu erwarten waren. Die Messungen wurden auf die Resonanzeinfänge, d. h. auf Elektronenenergien kleiner als $10 \mathrm{eV}$, beschränkt.

\section{Meßergebnisse}

Die Versuche wurden mit einem CH 3-Massenspektrometer und einer modifizierten TO 4-Festkörperionenquelle der A t la s - Werke, Bremen, durchgeführt. Die Alkalihalogenidproben wurden aus einem Graphittiegel in den Ionisierungsraum verdampft, durch Elektronen-

3 R. C. Schoonmaker u. R. F. Porter, J. Chem. Phys. 30, 283 [1959].

4 T.A. Milne u. H. M. Klein, J. Chem. Phys. 33, 1628 [1960]. 